Research Article

\title{
Use of Hydrocolloid Gums to Modify the Pasting, Thermal, Rheological, and Textural Properties of Sweet Potato Starch
}

\author{
Syed Ali Shahzad, Shahzad Hussain (D), Mohamed S. Alamri, Abdellatif A. Mohamed (D), \\ Ali S. Ahmed, Mohamed A. Ibraheem, and Akram A. Abdo Qasem
}

Department of Food Sciences, College of Food and Agricultural Sciences, King Saud University, P.O. Box 2460,
Riyadh 11451, Saudi Arabia

Correspondence should be addressed to Shahzad Hussain; shhussain@ksu.edu.sa

Received 30 April 2019; Revised 29 August 2019; Accepted 17 September 2019; Published 30 October 2019

Academic Editor: Victor H. Perez

Copyright (c) 2019 Syed Ali Shahzad et al. This is an open access article distributed under the Creative Commons Attribution License, which permits unrestricted use, distribution, and reproduction in any medium, provided the original work is properly cited.

\begin{abstract}
Effect of hydrocolloids (arabic, xanthan, cress seed, fenugreek, flaxseed, and okra gums) at 0.5 and $2.0 \%$ concentrations on sweet potato starch pasting, viscoelastic, textural, and thermal properties was studied. Rapid Visco Analyzer (RVA) measurements showed that the final viscosity was increased as a function of gum irrespective of their concentration except with cress seed gum. Dynamic rheological data revealed that the magnitude of the moduli was increased as a function of angular frequency and no crossovers were perceived among them. Consistency coefficient $(k)$ was decreased by the addition of any gum and also with increasing concentration of gums except with xanthan gum at $25^{\circ} \mathrm{C}$. Flow behavior index $(n)$ data showed that starch gum blends possessed more pseudoplastic shear thinning behavior than those for the control at $25^{\circ} \mathrm{C}$ and pseudoplasticity was further increased with increasing concentration of xanthan, fenugreek, flaxseed, and okra gums. Texture analysis data displayed that after overnight storage of starch gels at room temperature, hardness was significantly increased by the addition of gums except for xanthan. Differential scanning calorimeter (DSC) profile showed that enthalpy was decreased by the inclusion of xanthan gum.
\end{abstract}

\section{Introduction}

Sweet potato (Ipomoea batatas L.) is a tuber like a potato that belongs to the family Convolvulaceae, widely cultivated in China, Nigeria, and America. It is a rich source of starch as it accounts for $58-76 \%$ on a dry basis. The average size of the starch granule is about $18 \mu \mathrm{m}$ which is much smaller as compared to the potato starch $[1,2]$. Sweet potato starch is considered commercially important for many processed foods as it has been extensively utilized in bakery products, snack foods, starch noodles, and confectionery products [3]. However, most of the time, properties of the native starches are undesirable like high shear behavior which causes the disruption of the starch gel network, high tendency to retrograde during storage which causes the syneresis, and the inferior gelatinization stability during prolonged heating [4]. Therefore, the properties of native starches should be improved to make them suitable for food processing applica- tions. It can be done either physically or chemically to meet the specific requirement. From the safety point of view, the preferred method to produce the modified starch is that which involves no or very little chemical agents and produces minimum waste. Therefore, increase attention has been proposed on the utilization of nonstarch hydrocolloids to alter the pasting properties, gelatinization characteristics, and the retrogradation in starch-based products due to their accessibility, safety, and low cost $[5,6]$. Pasting, thermal, and rheological properties of the starch gum blends are largely dependent on the type, concentration, molecular weight, and structure of the hydrocolloids mixed with the starch. Appropriate hydrocolloid inclusion may change the enthalpy of gelatinization, reduce the firmness of the gel, and retard the retrogradation which reduces the syneresis [7]. It may also play a crucial role in enhancing or keeping the desirable textural characteristics and stabilizing the products over a prolonged storage [8]. Thus, starch/gum blends are valuable 
to be used in a vast range of food products. Recently, some researches have investigated the effect of hydrocolloids on the modification of sweet potato starch properties $[9,10]$.

Our aim of the study was to examine the effect of arabic, xanthan, garden cress seed, fenugreek, flaxseed, and okra gum at different concentrations and compare their effects on the rheological and functional properties of sweet potato starch.

\section{Material and Methods}

2.1. Materials. Commercial arabic and xanthan gums were obtained from Qualikems Fine Chem Pvt. Ltd. (India). The materials for isolation of starch and extraction of gum (sweet potatoes, garden cress, flaxseed, fenugreek, and okra pods) were purchased from a local supermarket. All types of analytical grade chemicals were obtained from Sigma, St. Louis, UK.

\subsection{Methods}

2.2.1. Isolation of Sweet Potato Starch. Sweet potatoes were washed thoroughly, peeled, and sliced into small pieces. Small pieces of sweet potato were mixed with distilled water at a ratio of $1: 1$, blended in a household blender for 3 minutes, and filtered through cheesecloth. Residues were resuspended in distilled water at a ratio of $1: 2$ and filtered. Starch was allowed to settle down at room temperature. After a few hours, the supernatant was discarded and starch was resuspended in distilled water and centrifuged at $3000 \mathrm{~g}$ for 10 minutes. The supernatant was discarded, and the top dark layer was removed. The centrifugation step was repeated until clean starch was obtained. Isolated starch was mixed with acetone and air-dried, ground to fine powder, sieved, and stored in an airtight glass bottle at $4^{\circ} \mathrm{C}$.

\subsection{Extraction of Gums}

2.3.1. Garden Cress Seed Gum. Seeds were cleaned from extraneous material and soaked $(100 \mathrm{~g})$ in $2 \mathrm{~L}$ of distilled water under gentle stirring for 3 hours. The filtrate was collected by squeezing swollen seeds with cheesecloth. Filtrate was freeze-dried $\left(-60^{\circ} \mathrm{C}\right.$, alpha $1,4 \mathrm{D}$ Plus, Martin Christ Gefriertrocknungsanlagen $\mathrm{GmbH}$, Germany), pulverized, and stored in an airtight glass bottle till further use.

2.3.2. Fenugreek Gum. Stones, dirt, and broken seeds were removed from fenugreek seeds and soaked (150 g) for overnight under continuous stirring in $2 \mathrm{~L}$ of distilled water. Ruptured seeds were removed by filtration, and ethanol was added to precipitate the extracted galactomannan. Ethanol addition was continued until the extracted galactomannan was obtained. Precipitated galactomannan was freeze-dried (as explained above), pulverized, and kept in an airtight glass bottle till further use.

2.3.3. Flaxseed Gum. Seeds were cleaned from foreign material, other seeds, and dust. The extraction was done as described by Qian et al. [11], where $500 \mathrm{~g}$ of flaxseeds was soaked overnight under continuous stirring in 4 liters of distilled water. The filtrate was collected by separating flaxseeds with cheesecloth. It was centrifuged, and the supernatant was collected. One volume of $100 \%$ ethanol was added in supernatant to precipitate the gum and recovered it by centrifugation. Precipitated gum was freeze-dried (as explained above), crushed, and kept in an airtight glass bottle till further use.

2.3.4. Okra Gum. Okra pods were washed and cut into small pieces, and seeds were removed. Okra gum was extracted according to Alamri [9], $200 \mathrm{~g}$ of seedless pods was blended for 5 minutes in a household blender in $0.05 \mathrm{M} \mathrm{NaOH}$ and centrifuged, and supernatant was collected after the $\mathrm{pH}$ was adjusted to neutral. Freeze-dried (as explained above) supernatant was milled and kept in an airtight glass bottle till further use.

2.4. Preparation of Starch Gum Blends. Starch/gum blends were constituted by substituting 0.5 or $2.0 \%$ of the starch with the gum of interest (arabic, xanthan, garden cress seed, fenugreek, flaxseed, and okra). During the whole analysis, plain sweet potato was used as a control. Distilled water was added to the blend of starch and gum to make slurry. The slurry was freeze-dried instead of hot air-dried to prevent starch gelatinization, and dried powder was stored in airtight jars.

2.5. Rapid Visco Analyzer Measurements. The pasting properties of plain starch and starch blends with gums were determined by a Rapid Visco Analyzer model 3D (Newport Scientific, Sydney, Australia), and data was analyzed by Thermocline (Newport Scientific, Sydney, Australia) software. A designed heating and cooling cycle was programmed. The rotation rate of the paddle was kept 960 RPM for the initial 10 seconds and then reduced to 160 RPM during the whole experiment. Control or starch/gum blends ( $3 \mathrm{~g}, 14 \%$ moisture basis) were weighed into a RVA aluminum canister, and the total weight $(28 \mathrm{~g})$ was completed by adding distilled water. The slurry was retained at $50^{\circ} \mathrm{C}$ for $30 \mathrm{sec}$, heated to $95^{\circ} \mathrm{C}$ (at $10.23^{\circ} \mathrm{C} /$ minute for $4.40 \mathrm{~min}$ ). The sample was kept at $95^{\circ} \mathrm{C}$ for 4 minutes and cooled to $50^{\circ} \mathrm{C}$ in $2 \mathrm{~min}$ at $10.23^{\circ} \mathrm{C} /$ minute, and finally, it was maintained at $50^{\circ} \mathrm{C}$ for $2 \mathrm{~min}$.

2.6. Dynamic Rheology and Rheological Behavior of Starch Gels. The gel obtained from the RVA test was used for the dynamic rheological testing by using a TA Discovery Hybrid Rheometer (New Castle, USA). The rheometer was equipped with a $\left(2^{\circ}\right)$ cone and plate system ( $40 \mathrm{~mm}$ in diameter), and the gap between the plates was adjusted to $100 \mu \mathrm{m}$. The excess gel was cleaned with a spatula and adjusted to the geometry gap. At 5\% constant strain, dynamic rheology data was recorded at $25^{\circ} \mathrm{C}$ with frequency sweeps ranging from 0.1 to $100 \mathrm{rad} / \mathrm{s}$. The data were analyzed using TA instrument software. The storage modulus $\left(G^{\prime}\right)$, loss modulus $\left(G^{\prime \prime}\right)$, and tangent $\left(\tan \delta=G^{\prime \prime} / G^{\prime}\right)$ were determined. The frequency range used here is typically used for the frequency sweep so that $G^{\prime}$ and $G^{\prime \prime}$ were within the linear region. The following equations were used to explain the experimental curves:

$$
\begin{aligned}
& G^{\prime}=K^{\prime} \omega^{n^{\prime}}, \\
& G^{\prime \prime}=K^{\prime \prime} \omega^{n^{\prime \prime}},
\end{aligned}
$$


TABLE 1: Effect of gums on pasting properties of sweet potato starch.

\begin{tabular}{|c|c|c|c|c|c|c|c|c|}
\hline Parameter & Conc. (\%) & Control & Arabic & Xanthan & Cress seed & Fenugreek & Flaxseed & Okra \\
\hline \multirow{2}{*}{ Peak viscosity $(\mathrm{mPa} \cdot \mathrm{s})^{\dagger}$} & 0.5 & $5603 \pm 28^{\mathrm{c}}$ & $4359 \pm 33^{\mathrm{e}}$ & $6217 \pm 16^{\mathrm{a}}$ & $5235 \pm 19^{\mathrm{d}}$ & $5815 \pm 25^{\mathrm{b}}$ & $5617 \pm 84^{\mathrm{c}}$ & $5594 \pm 77^{\mathrm{c}}$ \\
\hline & 2.0 & $5603 \pm 28^{\mathrm{b}}$ & $4265 \pm 20^{f}$ & $8719 \pm 74^{\mathrm{a}}$ & $4802 \pm 17^{\mathrm{e}}$ & $5516 \pm 40^{c}$ & $5238 \pm 10^{\mathrm{d}}$ & $5217 \pm 27^{\mathrm{d}}$ \\
\hline \multirow{2}{*}{ Breakdown viscosity $(\mathrm{mPa} \cdot \mathrm{s})$} & 0.5 & $2805 \pm 19^{\mathrm{b}}$ & $1636 \pm 11^{\mathrm{e}}$ & $3308 \pm 44^{\mathrm{a}}$ & $2751 \pm 49^{\mathrm{b}}$ & $2552 \pm 77^{\mathrm{c}}$ & $2432 \pm 43^{\mathrm{d}}$ & $2425 \pm 25^{\mathrm{d}}$ \\
\hline & 2.0 & $2805 \pm 19^{\mathrm{b}}$ & $1524 \pm 25^{\mathrm{e}}$ & $4666 \pm 68^{\mathrm{a}}$ & $2423 \pm 23^{\mathrm{d}}$ & $2766 \pm 22^{\mathrm{b}}$ & $2575 \pm 25^{\mathrm{c}}$ & $2558 \pm 41^{\mathrm{c}}$ \\
\hline \multirow{2}{*}{ Final viscosity (mPa.s) } & 0.5 & $3730 \pm 25^{\mathrm{e}}$ & & & & $4329 \pm 47^{\mathrm{a}}$ & $4175 \pm 25^{\mathrm{b}}$ & $4179 \pm 30^{\mathrm{b}}$ \\
\hline & 2.0 & $3730 \pm 25^{\mathrm{d}}$ & $3869 \pm 32^{c}$ & $4553 \pm 43^{\mathrm{a}}$ & $3429 \pm 40^{\mathrm{e}}$ & $3944 \pm 56^{\mathrm{b}}$ & $3854 \pm 47^{\mathrm{c}}$ & $3892 \pm 12^{\mathrm{bc}}$ \\
\hline \multirow{2}{*}{ Setback (mPass) } & 0.5 & $932 \pm 15^{\mathrm{e}}$ & & $930 \pm 5.6^{\mathrm{e}}$ & $1126 \pm 15^{\mathrm{b}}$ & $1066 \pm 33^{c}$ & $990 \pm 12^{\mathrm{d}}$ & $1010 \pm 27^{\mathrm{d}}$ \\
\hline & 2.0 & $932 \pm 15^{\mathrm{e}}$ & $1128 \pm 28^{\mathrm{b}}$ & $500 \pm 11^{\mathrm{f}}$ & $1050 \pm 39^{c}$ & $987 \pm 30^{\mathrm{d}}$ & $1191 \pm 40^{\mathrm{a}}$ & $1233 \pm 17^{\mathrm{a}}$ \\
\hline \multirow{2}{*}{ Pasting temp. $\left({ }^{\circ} \mathrm{C}\right)$} & 0.5 & $76.67 \pm 0.2^{\mathrm{b}}$ & $71.35 \pm 0.3^{\mathrm{f}}$ & $78.38 \pm 0.2^{\mathrm{a}}$ & $76.78 \pm 0.1^{\mathrm{b}}$ & $73.50 \pm 0.5^{\mathrm{d}}$ & $74.35 \pm 0.2^{\mathrm{c}}$ & $72.97 \pm 0.1^{\mathrm{e}}$ \\
\hline & 2.0 & $76.67 \pm 0.2^{\mathrm{b}}$ & $75.15 \pm 0.1^{\mathrm{c}}$ & $77.43 \pm 0.2^{\mathrm{a}}$ & $77.57 \pm 0.1^{\mathrm{a}}$ & $75.23 \pm 0.1^{\mathrm{c}}$ & $75.12 \pm 0.1^{c}$ & $75.10 \pm 0.2^{c}$ \\
\hline
\end{tabular}

Mean values with different superscripts within the same rows are significantly $(p<0.05)$ different. ${ }^{\dagger} \mathrm{mPa} \cdot \mathrm{s}=$ millipascal-second.

where $G^{\prime}$ is storage modulus, $G^{\prime \prime}$ is loss modulus, $K^{\prime}$ is the consistency index based on $G^{\prime}, K^{\prime \prime}$ is the consistency index based on $G^{\prime \prime}, n^{\prime}$ and $n^{\prime \prime}$ are flow behavior indices, and $\omega$ is angular frequency.

The flow curves as a dependent of shear stress on shear rate were determined at different temperatures $(25,35$, and $\left.45^{\circ} \mathrm{C}\right)$ with a shear rate of 0 to $200(1 / \mathrm{s})$ using the TA Discovery Hybrid Rheometer (New Castle, USA) equipped with a cone-plate measuring system $\left(2^{\circ}\right.$ angle, $40 \mathrm{~mm}$ in diameter). The experimental curves were described by the power law model:

$$
\tau=K \dot{\gamma}^{n}
$$

where $\tau$ is shear stress $(\mathrm{Pa}), K$ is consistency coefficient (Pa.s), $\dot{\gamma}$ is the shear rate $\left(\mathrm{s}^{-1}\right)$, and $n$ is the flow behavior index (dimensionless).

2.7. Gel Texture Analysis. RVA-cooked samples $(35 \mathrm{~mm}$ in height) were retained in aluminum canisters $(67.5 \mathrm{~mm}$ in height and $36 \mathrm{~mm}$ diameter) and stored overnight at room temperature. They were analyzed for hardness, cohesiveness, springiness, adhesiveness, and chewiness by using the Brookfield Texture Analyzer model CT3 (Brookfield Engineering Laboratories, Inc., Middleboro, USA). Every aluminum canisters were positioned upright on the metal plate of the texture analyzer. The test was composed of an insertion of the cylindrical probe $(35 \mathrm{~mm}$ high and $12.7 \mathrm{~mm}$ wide) up to $10 \mathrm{~mm}$ in two penetration cycles with a speed of $0.5 \mathrm{~mm} / \mathrm{se}$ cond. Data was collected directly from the screen.

2.8. Thermal Analysis. Plain starch and starch blends with gums (5-10 mg) were weighed in aluminum pans, and $10 \mu \mathrm{l}$ distilled water was added. Pans were hermetically sealed and allowed to equilibrate at room temperature for $2 \mathrm{hrs}$. The empty pan was used at the reference cell. Thermal scanning was done using Q2000 TA DSC (New Castle, USA). Samples were thermally scanned from 25 to $120^{\circ} \mathrm{C}$ at $10^{\circ} \mathrm{C} / \mathrm{min}$ heating rate. For each scan, the flowing thermal parameters were obtained, enthalpy $\Delta H(\mathrm{~J} / \mathrm{g})$, onset temperature $\left(T_{\mathrm{o}}\right)$, and peak temperature $\left(T_{\mathrm{p}}\right)$, using Universal Analysis Software.
2.9. Statistical Analysis. All data were collected in triplicate and analyzed using the ANOVA method. Multicomparison test Duncan's Multiple Range (DMR) at sig $\leq 0.05$ was used to compare means using SPSS (IBM Statistical Analysis Version 21). It allowed us to detect the significant effect of gums on sweet potato starch.

\section{Results and Discussions}

3.1. Rapid Visco Analyzer Measurements. Pasting properties of native sweet potato starch and sweet potato starch/gum blends are presented in Table 1. Pasting temperature (PT) showed the extent of delay in starch gelatinization. For the control, it was found to be $76.67^{\circ} \mathrm{C}$. It was significantly increased with xanthan irrespective of its concentration which is in agreement with Leite et al. [12] who evaluated the xanthan effect on cassava starch and observed a rise in the PT with xanthan. On the other hand, PT of arabic, fenugreek, flaxseed, and okra were significantly lower than that of the control regardless of their concentrations. The RVA data showed some similarity in the behavior of xanthan gum and cress seed gums with respect to PT as shown in Table 1. Although arabic gum exhibited the least PT, it had the highest setback among the other gums. The significant change in the peak viscosity (PV) with the addition of gum was observed. However, the change was dependent on the gum type and its concentration. According to some researchers, the PV signifies the point of maximum swelling of starch granule, the quantity of leached amylose, the competition among ungelatinized starch granules for free water, friction between swollen starch granules, and amylose lipid complex formation [13]. The PV of the control significantly decreased in the presence of arabic, cress seed, and okra gums regardless of their concentrations and also with $2.0 \%$ fenugreek and flaxseed gums. Addition of arabic gum caused the maximum reduction, i.e., 4359 and $4265 \mathrm{mPa} \cdot \mathrm{s}$, as compared to the control for 0.5 and $2.0 \%$ concentrations, respectively. This could be attributed to the gum ability to mask the surface of the starch granules, limit water absorption, and enhance granule-granule interactions which result in reduced swelling and limited increase in the PV $[14,15]$. The reduction in PV was higher at the higher gum concentration except for xanthan gum. The drop in PV was more 
pronounced for the cress seed where $0.5 \%$ reduced the PV of the control by $368 \mathrm{mPa} \cdot \mathrm{s}$ and with $2 \%$ by $800 \mathrm{mPa} \cdot \mathrm{s}$. The drop in PV could be attributed to the lack of synergistic association between the gum and leached amylose in the continuous phase which executed less control on the water molecules resulting in lower PV [16]. These results were consistent with $[9,15]$. Similar trends were noticed with other gums, but xanthan gum increased the $\mathrm{PV}$ by $614 \mathrm{mPa} \cdot \mathrm{s}$ and 3116 for the 0.5 and $2.0 \%$ concentrations, respectively. Similar findings were reported for Colocasia starch with xanthan gum [17]. Therefore, if we consider the starch system as biphasic, the effect of gum on the PV is predominantly due to how the gum interacts with the leached amylose in the continuous phase. The more effective gum is the one that interacts with amylose and with the excess water after starch granules reach their full size. Furthermore, swelling of granules reduced the accessibility of the continuous phase to gums which caused further raise in the paste viscosity [18, 19]. Another plausible explanation involves low molecular weight amylopectin interaction with gum molecules in the continuous phase [19]. Breakdown viscosity (BV) represents the drop in PV due to rupturing of starch granules and releasing of free water. Consequently, low (BV) values were obtained for blends compared with the control. According to Kaur et al. [20], reduction in BV could be due to the limited swelling of starch granules because of the presence of gum. Similar findings had been reported for tapioca and Colocasia starch with arabic gum $[14,16]$.

The final viscosity (FV) indicates the starch ability to form viscous paste after cooling to $50^{\circ} \mathrm{C}$ [21]. A significant increase in FV was observed for the blends regardless of the gum type or concentration except for cress seed. FV of native sweet potato starch was $3730 \mathrm{mPa} \cdot \mathrm{s}$ which increased up to $4553 \mathrm{mPa} \cdot \mathrm{s}$ with the addition of $2.0 \%$ xanthan gum. It might be due to the thickening effect of the gum that results in a raise in the viscosity of the continuous phase and overall suspension [17]. FV increased mainly due to the interaction between the gum and starch with the water released after granule rupture. Effect of xanthan was more pronounced as compared to the other gums. The different behaviors of xanthan gum were ascribed to the distinctive molecular structures and the flexibility of the gum molecular chains [22]. This behavior of xanthan gum could be due to lack of interaction with amylose which allows the formation of the strong network leading to higher FV. The same results were reported by [17]. On the contrary, cress seed exhibited the least FV compared to the control which is consistent with its behavior regarding the other RVA parameters or possibly its ability to interact with amylose and prevent its retrogradation.

Reassociation of dispersed starch molecules, swollen granules, and granular fragments was termed as a setback (SB) viscosity. It indicates amylose retrogradation, and the texture of starch-containing products was directly related to SB [21]. The substantial decrease in the SB value by $500 \mathrm{mPa} \cdot \mathrm{s}$ was observed for the $2.0 \%$ xanthan gum, but the $0.5 \%$ blend was not statistically different as compared to the control at $5 \%$ significant level (Table 1). The drop in the $\mathrm{SB}$ value was ascribed to the intermolecular association between the amylose and xanthan which caused a reduction in the occurrences of amylose-amylose interaction (retrogradation) during cooling. At higher xanthan concentration, the xanthan-amylose association seems to appear with more intensity which results in a dramatic decrease in the SB [12]. The results were positively correlated with the finding of Leite et al. [12] and Yadav et al. [17] who studied the effect of xanthan on cassava and Colocasia starch, respectively. In contrast, a significant increase was noticed in SB with all other gums. It might be due to the lack of interaction between the gums and amylose which resulted in amylose retrogradation causing higher SB as compared to the control [9]. SB was expected to decrease due to the absence of starch, but it increased instead which indicates that gums played a role in facilitating for amylose to retrograde. These results were consistent with the effect of gum on PV as tabulated in Table 1.

3.2. Dynamic Rheology and Rheological Behavior of Starch Gels. Variations in the storage modulus $\left(G^{\prime}\right)$, loss modulus $\left(G^{\prime \prime}\right)$, and the dynamic mechanical loss tangent ( $\tan \delta=$ $\mathrm{G}^{\prime \prime} / \mathrm{G}^{\prime}$ ) of sweet potato starch and blends as a function of frequency are illustrated in Figures 1 and 2. $G^{\prime}$ was well above than $G^{\prime \prime}$ over the whole frequency range $(0.1-100 \mathrm{rad} / \mathrm{sec})$ with little frequency dependence. The magnitude of $G^{\prime}$ and $G^{\prime \prime}$ was increased as a function of angular frequency, and no crossovers were observed among the moduli. It revealed the dominance of elastic behavior over viscosity. Furthermore, there was a strong relationship between starch and gums in the composite system where the greater angular frequency signifies the higher elasticity $\left(G^{\prime}>G^{\prime \prime}\right)$ and vice versa. Qiu et al. [23] classified such gels as weak gels. Similar results were reported by other authors $[12,24]$. A significant increase in storage and loss modulus was observed for cress seed, fenugreek, and flaxseed gums at both levels. It was also increased at a low concentration of okra gum and the higher concentration of xanthan gum. Increase in the moduli was ascribed to the increase in the viscoelastic behavior as the gums were concentrated in the continuous phase because of the thickening properties within the system [25]. So, it revealed the synergistic effect of the gum addition to the rheological properties of the starch which is apparent when we look at the $G^{\prime}$ of the control compared to the composites. This was in accordance with the previous findings that gum inclusion in starch increased the storage and loss modulus [15]. In contrast, it was decreased by the addition of arabic gum, a lower level of xanthan gum, and a higher level of okra gums. It could be due to the depolymerization of macromolecules because of the higher temperature [26]. Moreover, moduli were decreased by increasing concentration of arabic, cress seed, and okra gums. It could be ascribed to the uniform distribution of gums in the system at low concentration, while at higher levels, gum clustered itself in the composite system. Therefore, starch alone was competing for the available water which caused a decrease in the moduli at higher concentration.

The magnitude of dynamic mechanical loss tangent $(\tan \delta)$ was increased with increasing angular frequency. It is the ratio of $G^{\prime \prime}$ over $G^{\prime}$ which is an important rheological 

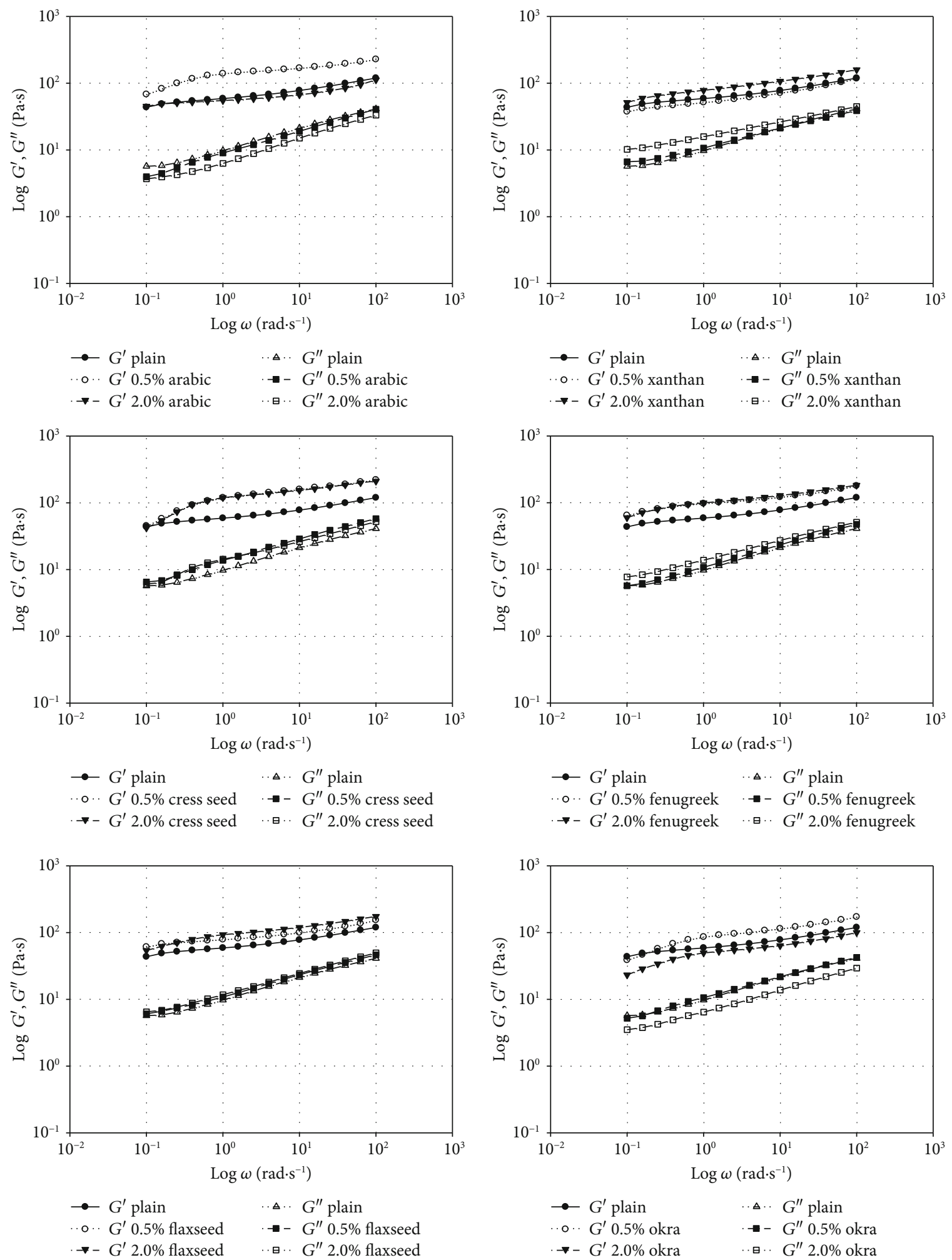

Figure 1: Plots of $\log G^{\prime}, G^{\prime \prime}$ versus $\log \omega$ of sweet potato starch gum blends at different gum concentrations.

parameter. According to Varela et al. [27], it shows the structural organization of viscoelastic material. Its value was decreased with the addition of gums as compared to the control except for xanthan. At lower frequencies, the $\tan \delta$ decreased for the control and the composites (Figure 2), but a steady increase was observed after that. However, at higher frequencies, $\tan \delta$ appeared to plateau. Lower $\tan (\delta)$ values indicated a more elastic gel with reference to the control. Furthermore, for all samples, its value was much smaller than 1.0 which indicates elastic behavior. Higher $\tan (\delta)$ values with 

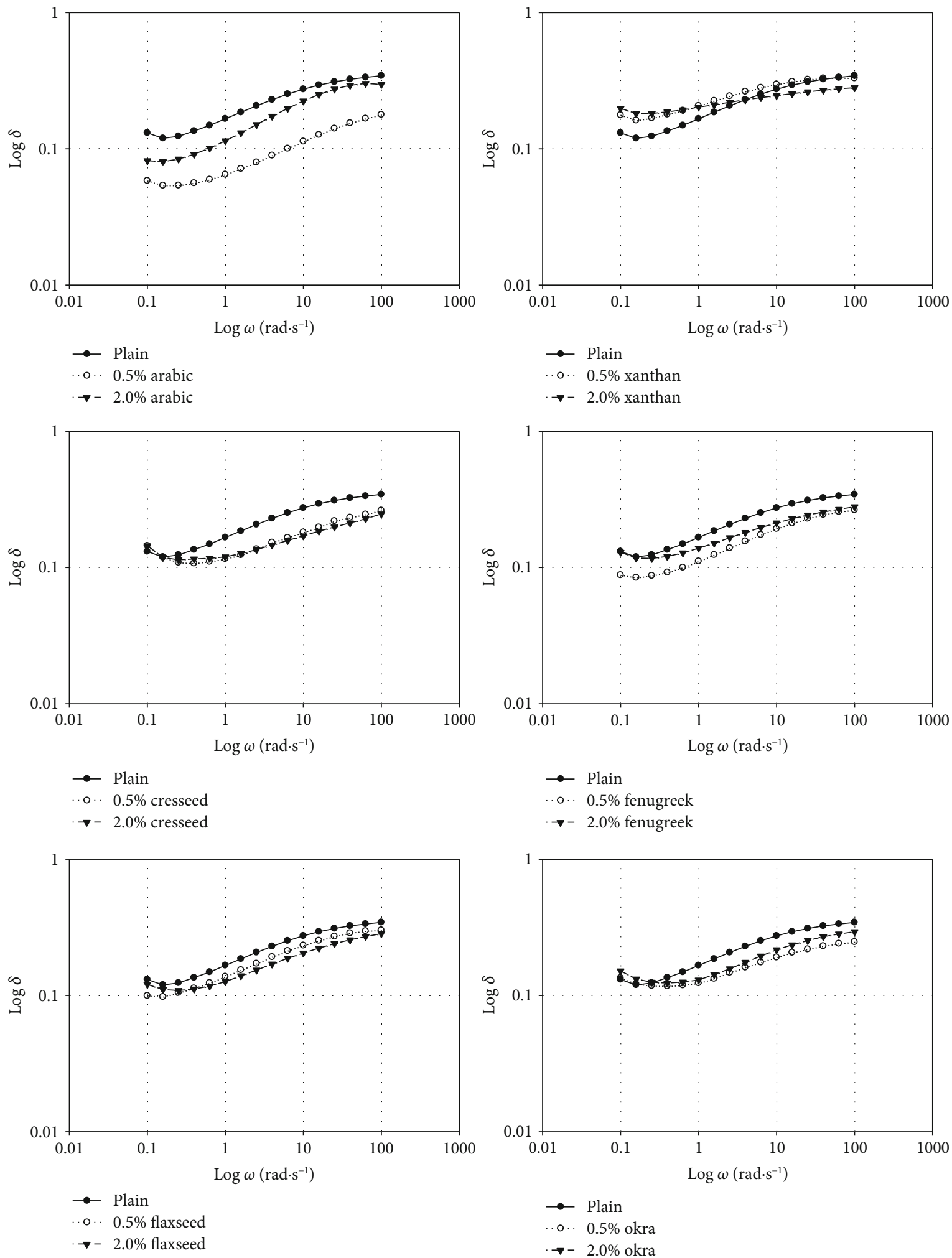

Figure 2: Plots of $\log \delta$ versus $\log \omega$ of sweet potato starch gum blends at different gum concentrations.

xanthan were noticed until 39.8 and $2.51 \mathrm{rad} / \mathrm{s}$ for $0.5 \%$ and $2.0 \%$ blends, respectively. It shows that the incorporation of xanthan caused the sweet potato starch to become more viscous. Similar results were reported by Toker et al. [28] who evaluated the effect of xanthan on corn starch and stated that the gel became more viscous after xanthan incorporation. It was attributed to the association between gum and amylose which hindered amylose retrogradation and limited junction zone development [29]. Another plausible explanation was the decrease in the concentration of leached amylose content 
TABLe 2: Effect of gums on $K^{\prime}, K^{\prime \prime}, n^{\prime}$, and $n^{\prime \prime}$ as determined from equations (1) and (2).

\begin{tabular}{lccccccc}
\hline Gum type & Gum (\%) & $K^{\prime}$ & $n^{\prime}$ & $R^{2}$ & $K^{\prime \prime}$ & $n^{\prime \prime}$ & $R^{2}$ \\
\hline Control & - & 1.774 & 0.134 & 0.98 & 1.007 & 0.306 & 0.99 \\
Arabic & $\mathbf{0 . 5}$ & 2.085 & 0.144 & 0.91 & 0.941 & 0.337 & 0.99 \\
& $\mathbf{2 . 0}$ & 1.747 & 0.110 & 0.92 & 0.833 & 0.338 & 0.99 \\
Xanthan & $\mathbf{0 . 5}$ & 1.720 & 0.152 & 0.98 & 1.046 & 0.272 & 0.99 \\
& $\mathbf{2 . 0}$ & 1.887 & 0.149 & 0.99 & 1.203 & 0.217 & 0.99 \\
Cress seed & $\mathbf{0 . 5}$ & 1.997 & 0.196 & 0.90 & 1.126 & 0.325 & 0.99 \\
& $\mathbf{2 . 0}$ & 1.985 & 0.196 & 0.88 & 1.116 & 0.306 & 0.99 \\
Fenugreek & $\mathbf{0 . 5}$ & 1.972 & 0.127 & 0.98 & 1.043 & 0.318 & 0.99 \\
& $\mathbf{2 . 0}$ & 1.974 & 0.141 & 0.98 & 1.145 & 0.282 & 0.99 \\
Flaxseed & $\mathbf{0 . 5}$ & 1.902 & 0.118 & 0.97 & 1.054 & 0.307 & 0.99 \\
& $\mathbf{2 . 0}$ & 1.935 & 0.151 & 0.98 & 1.078 & 0.307 & 0.99 \\
Okra & $\mathbf{0 . 5}$ & 1.874 & 0.188 & 0.95 & 1.021 & 0.309 & 0.99 \\
& $\mathbf{2 . 0}$ & 1.638 & 0.180 & 0.95 & 0.821 & 0.317 & 0.99 \\
\hline
\end{tabular}

with xanthan incorporation which decreases amylose retrogradation [10]. This was observed on the effect of xanthan gum on the setback discussed above.

$K^{\prime}$ and $K^{\prime \prime}$ or $n^{\prime}$ and $n^{\prime \prime}$ were calculated from equations (1) and (2) by subjecting the data (log of dynamic rheology) to linear regression, and results were tabulated in Table 2. $K$ and $n$ indicates the intercept and slope of the lines, respectively, for both $K^{\prime}$ and $K^{\prime \prime}$ or $n^{\prime}$ and $n^{\prime \prime}$ that represent $G^{\prime}$ and $G^{\prime \prime}$. In addition, $K^{\prime}$ represents the consistency index while $n^{\prime}$ indicates the shear behavior index of the power law model [30]. $K^{\prime}$ is nearly 2 -folds higher than $K^{\prime \prime}$, and both were increased by the addition of cress seed, fenugreek, and flaxseed gums irrespective of their concentrations and also with the lower concentration of okra and a higher concentration of xanthan gum. Similar results of an increase in $k^{\prime}$ values were reported by Shaari et al. [31] who studied the effect of xanthan and carrageenan gum on native corn starch. In contrast, they ( $K^{\prime}$ and $\left.K^{\prime \prime}\right)$ were reduced by the incorporation of the lower level of xanthan gum and a higher level of arabic and okra gums showing that elastic properties of sweet potato starch were reduced with the increasing concentration of these gums. It could be due to the interruption of the amylose network because these gums might prevent the gel formation.

The slope of weak gel is supposed to be positive while for true gel, it should be zero [32]. The results revealed that $R^{2}$ in the range of 0.88-0.99 and slopes of $\log G^{\prime}$ and $\log G^{\prime \prime}$ for all samples were positive $\left(n^{\prime}=0.110-0.196 ; n^{\prime \prime}=0.217-0.338\right)$ that gives the characteristics of typical weak gels. Inclusion of xanthan, cress seed, okra, low concentration of arabic, and a higher concentration of fenugreek and flaxseed gums into sweet potato starch increases the $n^{\prime}$ values that indicate the higher vulnerability of the sweet potato starch gels to the applied stress and decrease in the viscous properties by the addition of these gums. Similar results of an increase in $n^{\prime}$ as a function of gum were reported by $[31,32]$. On contrary, $n^{\prime \prime}$ values acted differently with the incorporation of these gums as they increased by arabic, cress, flaxseed, and okra gum inclusion and decrease by the inclusion of xanthan regardless of their concentration and with a higher concentration of fenugreek gum.

Starch pastes were assessed to determine the rheological behavior of starch/gum dispersions as a function of the shear rate by applying the power law model. The shear rate and the shear stress data were well fitted to the power law with a high regression coefficient $\left(R^{2}>0.96\right.$, Table 3$)$. The consistency coefficient $(k)$ could be used as an indicator of gel viscosity. Composite samples exhibited lower $K$ values with the incorporation of gum except for xanthan gum. According to Alamri [9], the $K$ value was considerably affected by the presence of a large amount of polysaccharides in the liquid phase of the composite system. Similar findings were reported by Alamri [9] and Shrivastava et al. [16] who studied the effect of okra addition on sweet potato and arabic on Colocasia starch, respectively. In contrast, the $k$ value further increased at higher xanthan gum (22.49 and 26.50 for 0.5 and $2.0 \%$ levels, respectively). This could be attributable to the greater hydration capacity and thickening effect of the xanthan gum [33]. The effect of xanthan was consistent with the previous findings of different authors who reported the determinant role of xanthan on $k$ value of sweet potato starch [34, 35]. As expected, $K$ value dropped at higher temperatures.

The power law model is extensively used to describe the pseudoplastic (non-Newtonian) liquid flow properties. Starch pastes either alone or with gums possessed pseudoplastic shear thinning behavior since their values were less than 1 as indicated in Table 3. The low behavior index was decreased with the addition of gum and with increasing gum concentration except for arabic and cress seed. These findings reflect that starch gum blends exhibit more nonNewtonian behavior as the concentration of gum increased. The decrease in the flow behavior index was ascribed to the more structural breakdown due to shearing. These results were consistent with Choi and Yoo [34] and Alamri [9] who incorporated xanthan and okra with sweet potato starch. However, a remarkable drop in the flow behavior index was observed with $2.0 \%$ xanthan gum because it exhibited 0.32 compared to the control $(0.42)$ at $25^{\circ} \mathrm{C}$. The reason behind the increase in the pseudoplastic shear thinning behavior of xanthan was its rigidity, rod-like conformation, and high molecular weight [24]. Different authors also reported similar findings for xanthan with sweet potato and corn starch $[31,35]$. An increase in the flow behavior index was observed when the temperature was increased to 45 from $25^{\circ} \mathrm{C}$. In contrast, it decreased when the temperature was further increased to $65^{\circ} \mathrm{C}$ which was in agreement with the typical behavior of biomaterials, where they exhibit higher pseudoplasticity at higher temperature [9].

3.3. Gel Texture Analysis. Textural parameters of native sweet potato and composites are summarized in Table 4. Retrogradation refers to the amylose mainly and to some extends amylopectin transformation and association. It occurred during cooling and storage of starch gels which result in the development of double helices and junction zone that eventually results in the formation of an organized structure due 
TABLE 3: Effect of gums on consistency coefficient $(K)$ and flow behavior index $(n)$ of sweet potato starch measured at 25,35 , and $45^{\circ} \mathrm{C}$.

\begin{tabular}{|c|c|c|c|c|c|c|c|c|c|}
\hline Temperature & Parameter & Conc. (\%) & Control & Arabic & Xanthan & Cress seed & Fenugreek & Flaxseed & Okra \\
\hline \multirow{6}{*}{$25^{\circ} \mathrm{C}$} & \multirow{2}{*}{$K^{\dagger}$} & 0.5 & 21.03 & 16.34 & 22.49 & 13.72 & 20.89 & 21.00 & 13.16 \\
\hline & & 2.0 & 21.03 & 10.37 & 26.50 & 13.25 & 18.80 & 17.78 & 6.88 \\
\hline & \multirow{2}{*}{$n^{\ddagger}$} & 0.5 & 0.42 & 0.37 & 0.35 & 0.40 & 0.36 & 0.37 & 0.41 \\
\hline & & 2.0 & 0.42 & 0.41 & 0.32 & 0.41 & 0.34 & 0.36 & 0.38 \\
\hline & \multirow{2}{*}{$R^{2}$} & 0.5 & 0.99 & 0.97 & 0.99 & 0.99 & 0.99 & 0.99 & 0.99 \\
\hline & & 2.0 & 0.99 & 0.99 & 0.99 & 0.99 & 0.99 & 0.99 & 0.99 \\
\hline \multirow{6}{*}{$45^{\circ} \mathrm{C}$} & \multirow{2}{*}{$K$} & 0.5 & 8.45 & 7.33 & 11.41 & 6.41 & 10.00 & 9.66 & 6.16 \\
\hline & & 2.0 & 8.45 & 5.06 & 16.18 & 6.33 & 8.95 & 9.05 & 3.11 \\
\hline & \multirow{2}{*}{$n$} & 0.5 & 0.47 & 0.47 & 0.44 & 0.42 & 0.44 & 0.45 & 0.48 \\
\hline & & 2.0 & 0.47 & 0.48 & 0.38 & 0.47 & 0.41 & 0.45 & 0.48 \\
\hline & \multirow{2}{*}{$R^{2}$} & 0.5 & 0.99 & 0.99 & 0.99 & 0.99 & 0.99 & 0.99 & 0.99 \\
\hline & & 2.0 & 0.99 & 0.99 & 0.99 & 0.99 & 0.99 & 0.99 & 0.99 \\
\hline \multirow{6}{*}{$65^{\circ} \mathrm{C}$} & \multirow{2}{*}{ K } & 0.5 & 7.08 & 7.02 & 9.72 & 5.68 & 8.29 & 7.87 & 5.26 \\
\hline & & 2.0 & 7.08 & 4.51 & 14.18 & 5.32 & 7.98 & 8.22 & 2.83 \\
\hline & \multirow{2}{*}{$n$} & 0.5 & 0.43 & 0.40 & 0.43 & 0.41 & 0.40 & 0.42 & 0.43 \\
\hline & & 2.0 & 0.43 & 0.45 & 0.35 & 0.42 & 0.39 & 0.40 & 0.44 \\
\hline & \multirow{2}{*}{$R^{2}$} & 0.5 & 0.99 & 0.99 & 0.99 & 0.99 & 0.99 & 0.99 & 0.99 \\
\hline & & 2.0 & 0.99 & 0.99 & 0.99 & 0.99 & 0.99 & 0.99 & 0.99 \\
\hline
\end{tabular}

${ }^{\dagger} K=\mathrm{Pa} \cdot \mathrm{s}$; indices are obtained by fitting the data to power law $\tau=K \dot{\gamma}^{n}$ and ${ }^{*} n=$ flow behavior index (dimensionless).

TABLE 4: Effect of gums on the TPA of sweet potato starch.

\begin{tabular}{|c|c|c|c|c|c|c|c|c|}
\hline Param & onc. (\%) & Control & Arabic & Xanthan & Cress seed & Fenugreek & Flaxseed & Okra \\
\hline \multirow[b]{2}{*}{ Hardness (N) } & 0.5 & $0.76 \pm 0.06^{\mathrm{cd}}$ & $1.35 \pm 0.03^{\mathrm{a}}$ & $0.70 \pm 0.04^{\mathrm{d}}$ & $0.93 \pm 0.02^{\mathrm{b}}$ & $0.95 \pm 0.02^{\mathrm{b}}$ & $0.80 \pm 0.02^{\mathrm{c}}$ & $0.81 \pm 0.06^{\mathrm{c}}$ \\
\hline & 2.0 & $0.76 \pm 0.06^{\mathrm{d}}$ & $1.06 \pm 0.03^{\mathrm{a}}$ & $0.53 \pm 0.04^{\mathrm{e}}$ & $0.90 \pm 0.01^{b c}$ & $0.83 \pm 0.03^{\mathrm{cd}}$ & $0.99 \pm 0.06^{\mathrm{ab}}$ & $1.04 \pm 0.12^{\mathrm{a}}$ \\
\hline \multirow[b]{2}{*}{ Cohesiveness } & 0.5 & $0.58 \pm 0.02^{\mathrm{b}}$ & $0.66 \pm 0.02^{\mathrm{a}}$ & $0.56 \pm 0.02^{\mathrm{b}}$ & $0.70 \pm 0.01^{\mathrm{a}}$ & $0.60 \pm 0.03^{b}$ & $0.68 \pm 0.04^{\mathrm{a}}$ & $0.57 \pm 0.03^{\mathrm{b}}$ \\
\hline & 2.0 & $0.58 \pm 0.02^{\mathrm{c}}$ & $0.64 \pm 0.03^{\mathrm{b}}$ & $0.52 \pm 0.03^{\mathrm{d}}$ & $0.67 \pm 0.03^{\mathrm{b}}$ & $0.59 \pm 0.01^{\mathrm{c}}$ & $0.73 \pm 0.02^{\mathrm{a}}$ & $0.59 \pm 0.20^{c}$ \\
\hline \multirow{2}{*}{ Springiness (mm) } & 0.5 & $10.33 \pm 0.12^{\mathrm{b}}$ & $10.00 \pm 0.01^{\mathrm{c}}$ & $10.83 \pm 0.15^{\mathrm{a}}$ & $9.77 \pm 0.15^{\mathrm{d}}$ & $9.57 \pm 0.12^{\mathrm{e}}$ & $9.87 \pm 0.06^{\mathrm{cd}}$ & $10.27 \pm 0.06^{\mathrm{b}}$ \\
\hline & 2.0 & $10.33 \pm 0.12^{\mathrm{b}}$ & $10.20 \pm 0.00^{\mathrm{bc}}$ & $11.00 \pm 0.20^{\mathrm{a}}$ & $9.77 \pm 0.06^{\mathrm{d}}$ & $9.77 \pm 0.06^{\mathrm{d}}$ & $10.07 \pm 0.06^{\mathrm{c}}$ & $10.30 \pm 0.10^{\mathrm{b}}$ \\
\hline \multirow{2}{*}{ Adhesiveness (mJ) } & 0.5 & $0.47 \pm 0.06$ & $0.43 \pm 0.06$ & $0.43 \pm 0.06$ & $0.43 \pm 0.06$ & $0.37 \pm 0.06$ & $0.37 \pm 0.06$ & $0.47 \pm 0.06$ \\
\hline & 2.0 & & $0.47 \pm 0.06^{\mathrm{b}}$ & $0.37 \pm 0.06^{\mathrm{C}}$ & $0.46 \pm 0.02^{\mathrm{b}}$ & $0.33 \pm 0.06^{\mathrm{C}}$ & $0.33 \pm 0.06^{\mathrm{C}}$ & $0.67 \pm 0.06^{\mathrm{a}}$ \\
\hline \multirow{2}{*}{ Chewiness $(\mathrm{N} \cdot \mathrm{mm})$} & 0.5 & & $8.94 \pm 0.51^{\mathrm{a}}$ & $4.27 \pm 0.36^{\mathrm{d}}$ & $6.42 \pm 0.03^{\mathrm{b}}$ & $5.44 \pm 0.36^{\mathrm{c}}$ & $5.37 \pm 0.36^{\mathrm{c}}$ & $4.77 \pm 0.35^{\mathrm{cd}}$ \\
\hline & 2.0 & $4.54 \pm 0.16^{\mathrm{d}}$ & $6.91 \pm 0.40^{\mathrm{ab}}$ & $3.11 \pm 0.06^{\mathrm{e}}$ & $5.93 \pm 0.24^{c}$ & $4.76 \pm 0.24^{\mathrm{d}}$ & $7.34 \pm 0.62^{\mathrm{a}}$ & $6.33 \pm 0.65^{b c}$ \\
\hline
\end{tabular}

Mean values with different superscripts within the same rows are significantly $(p<0.05)$ different.

to the reassociation of leached amylose and some amylopectin [36]. The hardness of starch gels was highly dependent on amylose retrogradation, and it is used to assess the extent of retrogradation. The data showed that hardness was significantly increased $(p \leq 0.5)$ with the addition of gum except for xanthan. The effect of arabic gum was pronounced because it increased the hardness up to 1.35 and $1.06 \mathrm{~N}$ for 0.5 and $2.0 \%$ concentrations, respectively. In addition, hardness results were highly correlated with setback values shown in Table 1. Increase in hardness could be attributed to the development of hydrogen bonds between starch polymers and gum macromolecules directed to the gel firmness [37]. The increase in hardness with the addition of gum was in accordance with the previous studies that the hardness of starch gels increased with gum addition $[37,38]$. In contrast, a reducing trend was noticed with $2.0 \%$ xanthan while $0.5 \%$ xanthan blend value was less than the control, but statistically, it was not different. Results revealed that xanthan seems to produce softer gel and retard the retrogradation of sweet potato starch gel and decreased it to 0.70 and $0.53 \mathrm{~N}$ compared to the control $(0.76 \mathrm{~N})$. Recently, similar findings were reported by Matia-Merino et al. [39] who studied the effect of xanthan on native corn starch. According to Tang et al. [29], a decrease in hardness might be due to the interruption of the gel by interactions between leached amylose and the gums. Furthermore, it was observed that hardness was decreased with increasing xanthan gum concentration and its effect on gel properties and water holding capacity of the system [40].

Cohesiveness indicates the strength of bonds which includes a mutual effect of cohesive and adhesive forces along with elasticity and viscosity. Flaxseed blends exhibit the 
TABLE 5: Effect of gums on the DSC profile of sweet potato starch.

\begin{tabular}{|c|c|c|c|c|c|c|}
\hline \multirow{2}{*}{$\begin{array}{l}\text { Parameters } \\
\text { Conc. (\%) }\end{array}$} & \multicolumn{2}{|c|}{ Onset temperature $\left({ }^{\circ} \mathrm{C}\right)$} & \multicolumn{2}{|c|}{ Peak temperature $\left({ }^{\circ} \mathrm{C}\right)$} & \multicolumn{2}{|c|}{ Enthalpy (J/g) } \\
\hline & 0.5 & 2.0 & 0.5 & 2.0 & 0.5 & 2.0 \\
\hline Control & $70.00 \pm 0.57^{\mathrm{c}}$ & $70.00 \pm 0.57^{\mathrm{c}}$ & $76.98 \pm 0.27^{\mathrm{a}}$ & $76.98 \pm 0.27^{\mathrm{b}}$ & $15.54 \pm 0.39^{\mathrm{a}}$ & $15.54 \pm 0.39^{\mathrm{b}}$ \\
\hline Arabic & $68.10 \pm 0.20^{\mathrm{d}}$ & $69.58 \pm 0.40^{\mathrm{c}}$ & $75.15 \pm 0.37^{\mathrm{b}}$ & $75.81 \pm 0.24^{\mathrm{c}}$ & $14.80 \pm 0.54^{\mathrm{b}}$ & $14.78 \pm 0.38^{\mathrm{c}}$ \\
\hline Xanthan & $71.53 \pm 0.38^{\mathrm{b}}$ & $71.60 \pm 0.61^{b}$ & $77.18 \pm 0.30^{\mathrm{a}}$ & $77.33 \pm 0.45^{\mathrm{b}}$ & $14.00 \pm 0.19^{c}$ & $10.71 \pm 0.33^{\mathrm{d}}$ \\
\hline Cress seed & $71.61 \pm 0.21^{\mathrm{ab}}$ & $72.35 \pm 0.18^{\mathrm{b}}$ & $77.07 \pm 0.17^{\mathrm{a}}$ & $77.32 \pm 0.35^{\mathrm{b}}$ & $15.69 \pm 0.22^{\mathrm{a}}$ & $16.32 \pm 0.20^{\mathrm{a}}$ \\
\hline Fenugreek & $69.49 \pm 0.44^{\mathrm{c}}$ & $70.08 \pm 0.76^{\mathrm{c}}$ & $75.34 \pm 0.37^{\mathrm{b}}$ & $76.11 \pm 0.21^{\mathrm{c}}$ & $15.52 \pm 0.16^{\mathrm{a}}$ & $15.57 \pm 0.17^{\mathrm{b}}$ \\
\hline Flaxseed & $69.89 \pm 0.78^{c}$ & $69.95 \pm 0.67^{\mathrm{c}}$ & $75.48 \pm 0.41^{\mathrm{b}}$ & $76.07 \pm 0.15^{\mathrm{c}}$ & $14.86 \pm 0.32^{\mathrm{b}}$ & $14.70 \pm 0.71^{\mathrm{c}}$ \\
\hline Okra & $72.33 \pm 0.03^{\mathrm{a}}$ & $73.53 \pm 0.24^{\mathrm{a}}$ & $77.15 \pm 0.39^{\mathrm{a}}$ & $78.12 \pm 0.80^{\mathrm{a}}$ & $15.93 \pm 0.52^{\mathrm{a}}$ & $16.82 \pm 0.40^{\mathrm{a}}$ \\
\hline
\end{tabular}

Mean values with different superscripts within the same column are significantly $(p<0.05)$ different.

highest cohesiveness ( 0.68 and 0.73 ) while xanthan blend displayed the lowest cohesiveness ( 0.56 and 0.52$)$ irrespective of their concentrations. The elasticity of the food sample was indicated by the springiness of the gel during initial deformation and is measured as the quantity needed to damage the gel structure. When its value is low, it reveals a broken gel structure into small pieces; on the contrary, big pieces indicate that the springiness is high [41]. It was significantly decreased with arabic, cress seed, fenugreek, and flaxseed gum which could mean that slow development of polymer aggregates causes low springiness at more viscous regions [42]. The adhesiveness of okra was found to be significantly higher regardless of their concentration as compared to the control. Alamri [9] reported that the inclusion of okra gum in sweet potato starch did not change the adhesiveness of the gel while Matia-Merino et al. [39] reported a decrease in adhesiveness with the addition of xanthan and basil seed gum at $0.5 \%$ level in corn starch.

3.4. Thermal Analysis. Thermal properties of native sweet potato starch and composites are presented in Table 5. Incorporation of gums significantly affects the gelatinization parameters. All blends along with the control displayed a single endotherm peak during gelatinization. Onset and peak temperatures of native sweet potato starch were 70.00 and 76.98 , respectively, which is significantly decreased with the addition of $0.5 \%$ arabic gum. However, fenugreek and flaxseed blends irrespective of their concentration along with the higher concentration of arabic blend exhibited statistically the same onset and significantly lower peak temperatures than those of the control. In contrast, increasing trends were observed with xanthan, cress seed, and okra gum irrespective of their concentration, but peak temperature was not statistically different from the control. According to Huang et al. [43] and Varela et al. [27], the increase in the onset might be due to the interaction between gum and starch at the early stage of gelatinization or the gum might have delayed the movement of water towards starch granules because of competition between starch and gum. A similar trend was reported by different authors with sweet potato starch $[9,10]$.

Gelatinization enthalpy $(\Delta H)$ signifies the area under the curve. It significantly decreased with arabic, xanthan, and flaxseed blends regardless of their concentration. It could be ascribed to the hydrogen bonding between gums and leached amylose which limits the internal interactions among amylopectin and amylose [29]. Furthermore, the reduction in enthalpy also related to the restriction of amylopectin chain movement in the presence of gums which also competed for available water with amylose and amylopectin [44]. The results were consistent with Huang [6] and Hussain [38] who reported a decrease in the enthalpy of sweet potato and rice starch with the addition of locust bean and flaxseed gum, respectively. However, conflict was observed as gelatinization enthalpy was significantly increased only at the $2.0 \%$ gum concentration with cress and okra gum. The gelatinization enthalpy increased from 15.54 to 16.32 and 16.82 (J/g) with the inclusion of cress seed and okra gum, respectively. It could be attributed to the hydrophilic behavior of the gum that prevented the migration of water which led to rise in the required energy for gelatinization [27].

\section{Conclusion}

Pasting, rheological, textural, and thermal properties of sweet potato starch were greatly influenced by the incorporation of various gums. Pasting properties revealed that setback viscosities of sweet potato starch/gum blend were increased as a function of gum irrespective of their concentration except for xanthan gum. Textural analysis results suggested that hardness was reduced only by the addition of xanthan gum, which reveals the inhibition of amylose retrogradation. Thermal analysis revealed that the enthalpy of gelatinization was increased by the addition of cress seed and okra gums due to the hydrophilic behavior of the gums which prevented the migration of water and led to the rise in the required energy for the gelatinization. Tan delta signified that the dominance of elastic properties over viscous ones and elastic character of starch/gum blends were further increased by the addition of gums except for xanthan gum. Keeping in view the objective of finding alternatives of arabic and xanthan gum, we can say that fenugreek gum could be used as xanthan replacement (with respect to peak viscosity) while cress could be used when low viscosity is needed. Although all gums had represented non-Newtonian behavior, okra and cress were more stable (less structural breakdown during shearing) than other gums. The results could be used for the development of sweet potato starch-based gelled deserts with different textures. 


\section{Data Availability}

Data used to support the findings of this study are included in the article.

\section{Conflicts of Interest}

The authors declare no conflict of interest.

\section{Acknowledgments}

The authors extend their appreciation to the Deanship of Scientific Research at King Saud University for funding this work through research group no. RGP-114.

\section{References}

[1] R. V. Kale, D. M. Shere, M. D. Sontakke, and K. S. Gadhe, "Effect of isolation methods on physicochemical and functional properties of sweet potato (Ipomoea batatas L.) starch," Journal of Pharmacognosy and Phytochemistry, vol. 6, no. 4, pp. 223-227, 2017.

[2] G. Lu, H. Huang, and D. Zhang, "Prediction of sweetpotato starch physiochemical quality and pasting properties using near-infrared reflectance spectroscopy," Food chemistry, vol. 94, no. 4, pp. 632-639, 2006.

[3] A. Gunaratne and H. Corke, "Functional properties of hydroxypropylated, cross-linked, and hydroxypropylated crosslinked tuber and root starches," Cereal Chemistry, vol. 84, no. 1, pp. 30-37, 2007.

[4] D. Gałkowska, M. Długosz, and L. Juszczak, "Effect of high methoxy pectin and sucrose on pasting, rheological, and textural properties of modified starch systems," Starch-Stärke, vol. 65 , no. 5-6, pp. 499-508, 2013.

[5] S. K. Baveja, K. V. R. Rao, and J. Arora, "Examination of natural gums and mucilages as sustaining materials in tablet dosage forms," Indian Journal of Pharmaceutical Sciences, vol. 50, no. 2, pp. 89-92, 1988.

[6] C.-C. Huang, "Physicochemical, pasting and thermal properties of tuber starches as modified by guar gum and locust bean gum," International Journal of Food Science \& Technology, vol. 44, no. 1, pp. 50-57, 2009.

[7] Q.-Q. Li, Y.-S. Wang, H.-H. Chen, S. Liu, and M. Li, "Retardant effect of sodium alginate on the retrogradation properties of normal cornstarch and anti-retrogradation mechanism," Food Hydrocolloids, vol. 69, pp. 1-9, 2017.

[8] T. Temsiripong, R. Pongsawatmanit, S. Ikeda, and K. Nishinari, "Influence of xyloglucan on gelatinization and retrogradation of tapioca starch," Food Hydrocolloids, vol. 19, no. 6, pp. 1054-1063, 2005.

[9] M. S. Alamri, "Sweet potato/potato starch and Abelmoschus esculentus-gum blends: thermal and textural properties," Starch - Stärke, vol. 66, no. 1-2, pp. 132-141, 2014.

[10] Z. Yu, Y.-S. Wang, H.-H. Chen, and Q.-Q. Li, "Effect of sodium alginate on the gelatinization and retrogradation properties of two tuber starches," Cereal Chemistry, vol. 95, no. 3, pp. 445455, 2018.

[11] K. Y. Qian, S. W. Cui, Y. Wu, and H. D. Goff, "Flaxseed gum from flaxseed hulls: extraction, fractionation, and characterization," Food Hydrocolloids, vol. 28, no. 2, pp. 275-283, 2012.
[12] T. D. Leite, J. F. Nicoleti, A. L. B. Penna, and C. M. L. Franco, "Effect of addition of different hydrocolloids on pasting, thermal, and rheological properties of cassava starch," Food Science and Technology, vol. 32, no. 3, pp. 579-587, 2012.

[13] S. Qiu, M. P. Yadav, Y. Liu, H. Chen, E. Tatsumi, and L. Yin, "Effects of corn fiber gum with different molecular weights on the gelatinization behaviors of corn and wheat starch," Food Hydrocolloids, vol. 53, pp. 180-186, 2016.

[14] H.-m. Chen, X. Fu, and Z.-g. Luo, "Effect of gum arabic on freeze-thaw stability, pasting and rheological properties of tapioca starch and its derivatives," Food Hydrocolloids, vol. 51, pp. 355-360, 2015.

[15] A. Singh, D. J. Geveke, and M. P. Yadav, "Improvement of rheological, thermal and functional properties of tapioca starch by using gum arabic," $L W T$, vol. 80, pp. 155-162, 2017.

[16] M. Shrivastava, R. B. Yadav, B. S. Yadav, and N. Dangi, "Effect of incorporation of hydrocolloids on the physicochemical, pasting and rheological properties of Colocasia starch," Journal of Food Measurement and Characterization, vol. 12, no. 2, pp. 1177-1185, 2018.

[17] K. Yadav, B. S. Yadav, R. B. Yadav, and N. Dangi, "Physicochemical, pasting and rheological properties of Colocasia starch as influenced by the addition of guar gum and xanthan gum," Journal of Food Measurement and Characterization, vol. 12, no. 4, pp. 2666-2676, 2018.

[18] D.-S. Sun and B. Yoo, "Rheological and thermal properties of blend systems of rice flour and potato starch," Food Science and Biotechnology, vol. 20, no. 6, pp. 1679-1684, 2011.

[19] M. J. Correa, C. Ferrero, C. Puppo, and C. Brites, "Rheological properties of rice-locust bean gum gels from different rice varieties," Food Hydrocolloids, vol. 31, no. 2, pp. 383391, 2013.

[20] L. Kaur, J. Singh, H. Singh, and O. J. McCarthy, "Starch-cassia gum interactions: a microstructure - rheology study," Food Chemistry, vol. 111, no. 1, pp. 1-10, 2008.

[21] A. Nawab, F. Alam, M. A. Haq, and A. Hasnain, "Effect of guar and xanthan gums on functional properties of mango (Mangifera indica) kernel starch," International Journal of Biological Macromolecules, vol. 93, Part A, pp. 630-635, 2016.

[22] P. Achayuthakan and M. Suphantharika, "Pasting and rheological properties of waxy corn starch as affected by guar gum and xanthan gum," Carbohydrate Polymers, vol. 71, no. 1, pp. 9-17, 2008.

[23] S. Qiu, M. P. Yadav, H. Chen, Y. Liu, E. Tatsumi, and L. Yin, "Effects of corn fiber gum (CFG) on the pasting and thermal behaviors of maize starch," Carbohydrate polymers, vol. 115, pp. 246-252, 2015.

[24] C. Kim and B. Yoo, "Rheological properties of rice starchxanthan gum mixtures," Journal of Food Engineering, vol. 75, no. 1, pp. 120-128, 2006.

[25] M. Alloncle and J.-L. Doublier, "Viscoelastic properties of maize starch/hydrocolloid pastes and gels," Food Hydrocolloids, vol. 5, no. 5, pp. 455-467, 1991.

[26] D. D. Warner and O. E. Araujo, "Kinetics of rheological properties of acacia solutions," Journal of Pharmaceutical Sciences, vol. 60, no. 6, pp. 863-866, 1971.

[27] M. S. Varela, A. S. Navarro, and D. K. Yamul, "Effect of hydrocolloids on the properties of wheat/potato starch mixtures," Starch-Stärke, vol. 68, no. 7-8, pp. 753-761, 2016. 
[28] O. S. Toker, M. Dogan, E. Canıylmaz, N. B. Ersöz, and Y. Kaya, "The effects of different gums and their interactions on the rheological properties of a dairy dessert: a mixture design approach," Food and Bioprocess Technology, vol. 6, no. 4, pp. 896-908, 2013.

[29] M. Tang, Y. Hong, Z. Gu, Y. Zhang, and X. Cai, "The effect of xanthan on short and long-term retrogradation of rice starch," Starch-Stärke, vol. 65, no. 7-8, pp. 702-708, 2013.

[30] A. A. Mohamed, M. S. Alamri, S. Hussain, M. A. R. Ibraheem, and A. A. QASEM, "Rheological properties of sweet potato starch-date syrup gel," Food Science and Technology, 2019.

[31] N. A. Shaari, R. Sulaiman, and C. Y. Cheok, "Rheological properties of native and modified corn starches in the presence of hydrocolloids," International Food Research Journal, vol. 24, no. 5, 2017.

[32] D. W. Choi and Y.-H. Chang, "Steady and dynamic shear rheological properties of buckwheat starch-galactomannan mixtures," Preventive Nutrition and Food Science, vol. 17, no. 3, pp. 192-196, 2012.

[33] B. Wang, L.-J. Wang, D. Li, N. Özkan, S.-J. Li, and Z.-H. Mao, "Rheological properties of waxy maize starch and xanthan gum mixtures in the presence of sucrose," Carbohydrate Polymers, vol. 77, no. 3, pp. 472-481, 2009.

[34] H. M. Choi and B. Yoo, "Steady and dynamic shear rheology of sweet potato starch-xanthan gum mixtures," Food Chemistry, vol. 116, no. 3, pp. 638-643, 2009.

[35] D.-D. Kim and B. Yoo, "Rheological behaviors of hydroxypropylated sweet potato starches influenced by guar, locust bean, and xanthan gums," Starch-Stärke, vol. 62, no. 11, pp. 584$591,2010$.

[36] M. Krystyjan, G. Adamczyk, M. Sikora, and P. Tomasik, "Long-term storage stability of selected potato starch - nonstarchy hydrocolloid binary gels," Food Hydrocolloids, vol. 31, no. 2, pp. 270-276, 2013.

[37] D. Gałkowska, K. Pycia, L. Juszczak, and P. Pająk, "Influence of cassia gum on rheological and textural properties of native potato and corn starch," Starch-Stärke, vol. 66, no. 11-12, pp. 1060-1070, 2014.

[38] S. Hussain, "Native rice starch and linseed gum blends: effect on the pasting, thermal and rheological properties," Czech Journal of Food Sciences, vol. 33, no. 6, pp. 556-563, 2015.

[39] L. Matia-Merino, M. Prieto, L. Roman, and M. Gómez, “The impact of basil seed gum on native and pregelatinized corn flour and starch gel properties," Food Hydrocolloids, vol. 89, pp. 122-130, 2019.

[40] M. Feng, X. Yang, J. Sun, X. Xu, and G. Zhou, "Study on retrogradation of maize starch-flaxseed gum mixture under various storage temperatures," International Journal of Food Science \& Technology, vol. 53, no. 5, pp. 1287-1293, 2018.

[41] Y. Phimolsiripol, U. Siripatrawan, and C. J. K. Henry, "Pasting behaviour, textural properties and freeze-thaw stability of wheat flour-crude malva nut (Scaphium scaphigerum) gum system," Journal of Food Engineering, vol. 105, no. 3, pp. 557-562, 2011.

[42] M. S. Alamri, A. A. Mohamed, S. Hussain, and H. A. Almania, "Legume starches and okra (Abelmoschus esculentus) gum blends: pasting, thermal, and viscous properties," Food Science and Technology Research, vol. 19, no. 3, pp. 381-392, 2013.
[43] C.-C. Huang, P. Lai, I.-H. Chen, Y.-F. Liu, and C.-C. R. Wang, "Effects of mucilage on the thermal and pasting properties of yam, taro, and sweet potato starches," LWT-Food Science and Technology, vol. 43, no. 6, pp. 849-855, 2010.

[44] M. H. Lee, M. H. Baek, D. S. Cha, H. J. Park, and S. T. Lim, "Freeze-thaw stabilization of sweet potato starch gel by polysaccharide gums," Food Hydrocolloids, vol. 16, no. 4, pp. 345-352, 2002. 


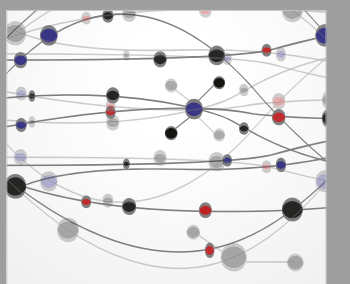

The Scientific World Journal
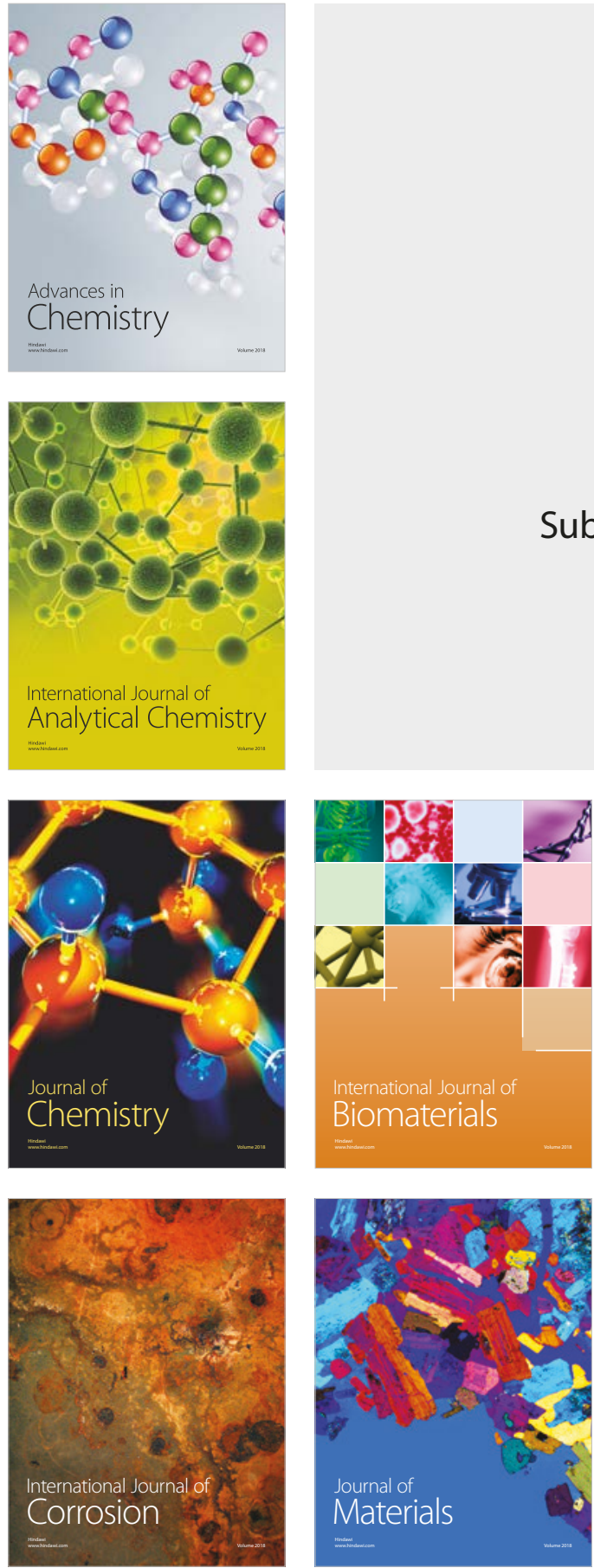

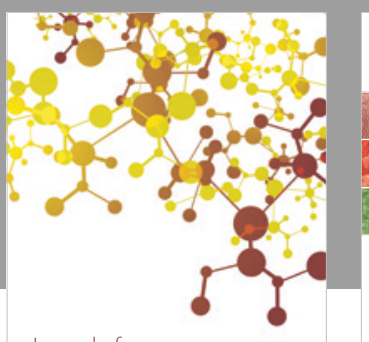

Journal of

Applied Chemistry
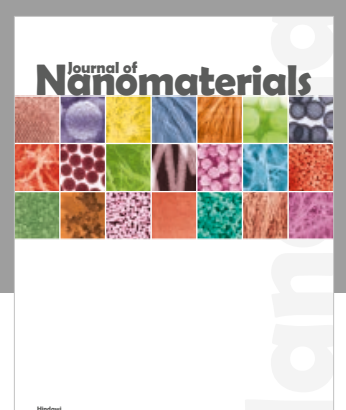

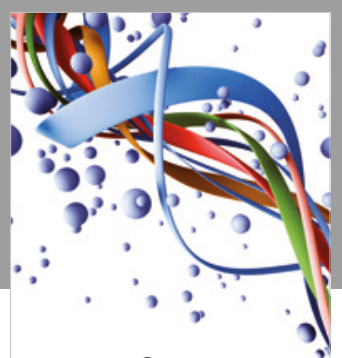

Scientifica

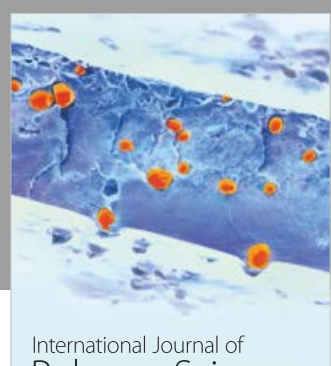

Polymer Science

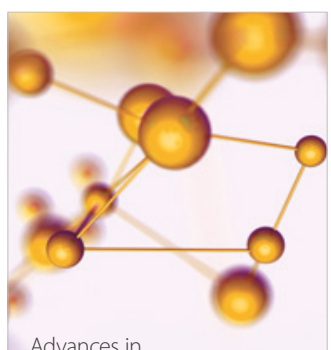

Physical Chemistry
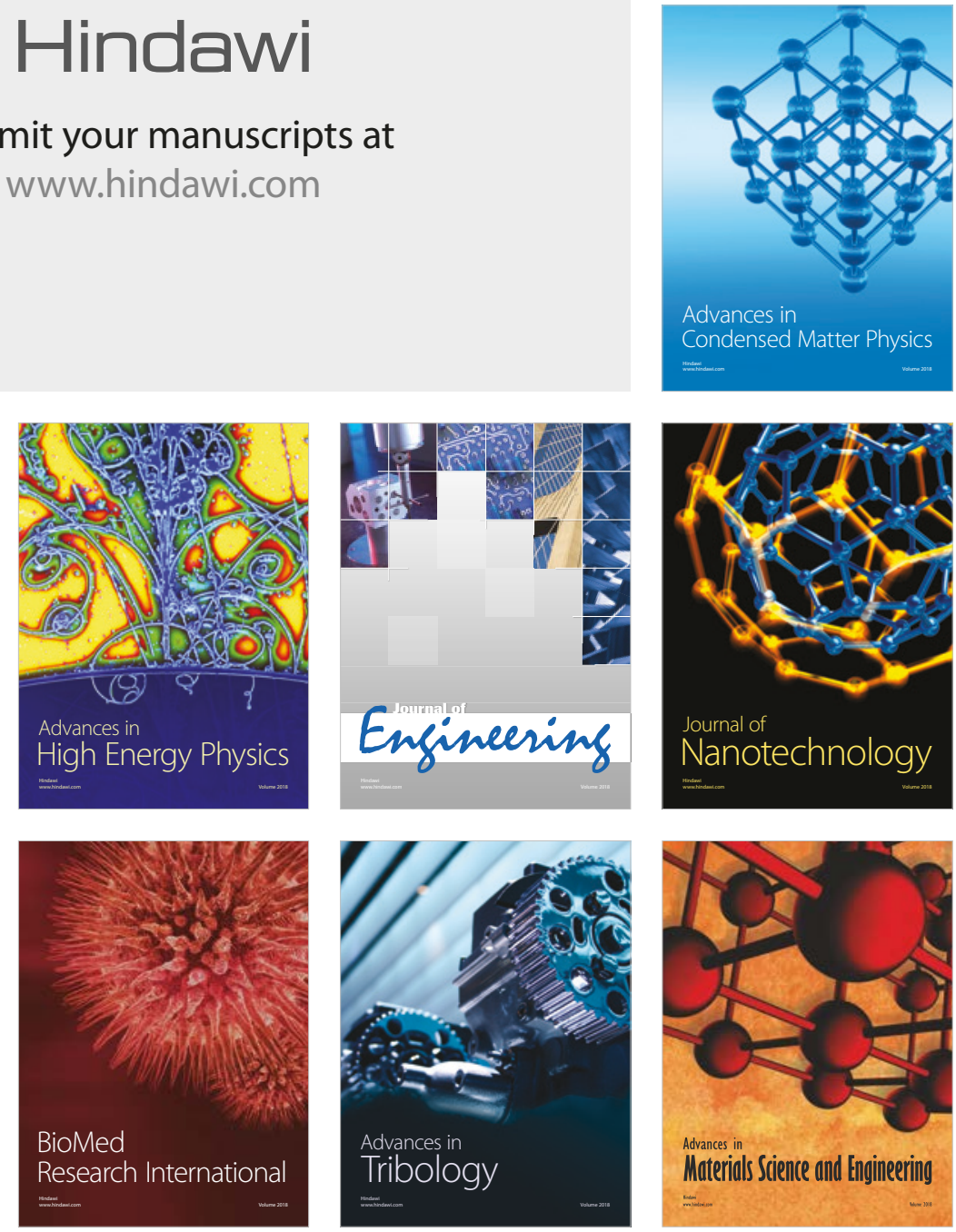